\title{
The Relationship of Percent Body Fat by Bioelectrical Impedance Analysis with Blood Pressure, and Glucose and Lipid Parameters
}

\author{
Junji Kobayashi ${ }^{1}$, Shunichi Murano ${ }^{2}$, Isao Kawamura ${ }^{3}$, Fumie Nakamura ${ }^{2}$, Yuko Murase ${ }^{1}$, \\ Masa-aki Kawashiri ${ }^{4}$, Atsushi Nohara ${ }^{5}$, Akimichi Asano ${ }^{4}$, Akihiro Inazu ${ }^{6}$, and Hiroshi Mabuchi ${ }^{5}$ \\ ${ }^{1}$ Department of Lifestyle-related Disease, Kanazawa University Graduate School of Medical Science, Kanazawa, Japan. \\ ${ }^{2}$ Department of Internal Medicine, Shimotsuga General Hospital, Tochigi, Japan. \\ ${ }^{3}$ Department of Surgery Shimotsuga General Hospital, Tochigi, Japan. \\ ${ }^{4}$ Department of Internal Medicine, Kanazawa University Graduate School of Medical Science, Kanazawa, Japan. \\ ${ }^{5}$ Division of Lipid Research, Kanazawa University Graduate School of Medical Science, Kanazawa, Japan. \\ ${ }^{6}$ Kanazawa University, Faculty of Medicine, School of Health Science, Laboratory Sciences, Kanazawa, Japan.
}

\begin{abstract}
The objective of this study was to clarify the clinical significance and usefulness of measuring percent body fat $(\mathrm{PBF})$ when compared with body mass index (BMI) in the Japanese population. A total of 2,483 Japanese individuals (1,380 men and 1,103 women) who underwent a medical checkup from 1999-2002 were employed. PBF was determined using bioelectrical impedance analysis (BIA). Relationships of age, BMI and PBF with several metabolic parameters, including blood pressure, lipids and plasma glucose levels were assessed in both genders separately. In men, PBF was a stronger determinant of total cholesterol (TC), low-density lipoprotein-cholesterol (LDL-C) and triglycerides (TG) compared with age and BMI, whereas in women, age was the strongest determinant of TC and LDL-C. In both genders, BMI was the strongest determinant of serum HDL-C among age, PBF and BMI. Based on these data, we suggest that measuring PBF by BIA is superior to BMI for predicting TC, LDL-C and TG in Japanese men.
\end{abstract}

J Atheroscler Thromb, 2006; 13:221-226.

Key words; Percent body fat, Bioelectrical impedance analysis, Body mass index, Plasma lipids

\section{Introduction}

Obesity is determined based on an individual's BMI, which is defined as body weight $(\mathrm{kg})$ divided by squared body height $\left(\mathrm{m}^{2}\right)$. The use of bioelectrical impedance analysis (BIA) for determining percent body fat $(\mathrm{PBF})$ is widely accepted as a safe, rapid, low cost and reliable technique ${ }^{1-3)}$. This method, as is the case in BMI, does not provide information on body fat distribution, which is in contrast to, such as $\mathrm{CT}^{4,5)}, \mathrm{MRI}^{6}$ ) and ultrasonography ${ }^{7)}$. Nevertheless, PBF determined by BIA, as in the case with BMI, is widely used in Ja-

Address for correspondence: Junji Kobayashi, Department of Lifestyle-related Disease Kanazawa University Graduate School of Medical Science Takara-machi 13-1, Kanazawa 920-8640, Japan.

E-mail: junji@med.kanazawa-u.ac.jp

Received: February 3, 2006

Accepted for publication: July 20, 2006 pan for screening an individual's body fat mass in medical checkups because of its simpleness; however, the clinical significance of measuring PBF has not been well studied in detail.

With this background, the aim of this study was to clarify the usefulness and clinical significance of measuring PBF by analyzing the relationship of $\mathrm{PBF}$ with several metabolic parameters, including blood pressure, plasma glucose, and plasma lipid levels in 2,483 Japanese individuals (1,380 men and 1,103 women) who underwent medical checkups from 1999-2002 in our department.

\section{Materials and Methods}

Table 1 shows the clinical profiles of the study subjects.

For individuals who underwent a medical checkup more than once during this period, the latest data 
Table 1. Profile of the study subjects

\begin{tabular}{lccc}
\hline & men & women & $p$ \\
\hline $\mathrm{n}$ & 1,380 & 1,103 & $\mathrm{~ns}$ \\
age, yr & $52.4 \pm 9.7$ & $52.4 \pm 9.5$ & $<0.0001$ \\
body mass index, $\mathrm{kg} / \mathrm{m}^{2}$ & $23.7 \pm 3.0$ & $22.8 \pm 3.1$ & $<0.0001$ \\
percent body fat, \% & $23.1 \pm 5.4$ & $29.4 \pm 6.2$ & $<0.0001$ \\
systolic BP, mmHg & $123.7 \pm 16.5$ & $119 \pm 17.6$ & $<0.0001$ \\
diastolic BP, mmHg & $80.6 \pm 10.8$ & $75.2 \pm 23.6$ & $<0.0001$ \\
fasting plasma glucose, mg/dL & $99.8 \pm 11.1$ & $94.5 \pm 9.8$ & $<0.001$ \\
HbAlc, \% & $4.98 \pm 0.37$ & $4.94 \pm 0.35$ & $<0.0001$ \\
total cholesterol, $\mathrm{mg} / \mathrm{dL}$ & $200 \pm 33.5$ & $208 \pm 34.3$ & $<0.0001$ \\
triglycerides, $\mathrm{mg} / \mathrm{dL}$ & $129 \pm 66.3$ & $94.3 \pm 57.1$ & $<0.0001$ \\
HDL-cholesterol, $\mathrm{mg} / \mathrm{dL}$ & $55.4 \pm 14.6$ & $67.1 \pm 16.2$ & $\mathrm{~ns}$ \\
LDL-cholesterol, mg/dL & $120 \pm 31.2$ & $122 \pm 30.9$ & $<0.0001$ \\
white blood cell, $\times 10^{3}$ & $5.9 \pm 1.7$ & $5.1 \pm 1.4$ & $<0.0001$ \\
red blood cell, $\times 10^{6}$ & $4.83 \pm 0.39$ & $4.4 \pm 0.3$ & $<0.0001$ \\
Hb, g/dL & $15.1 \pm 1.1$ & $13 \pm 1.2$ & \\
\hline
\end{tabular}

$\mathrm{BP}$, blood pressure; $\mathrm{Hb}$, hemoglobin

Values are shown as the mean \pm sd.

were used for this study. Individuals with AST $>100$, ALT $>100$, Cre $>1.5, \mathrm{HbA} 1 \mathrm{c}>6.5 \%, \mathrm{TG}>400 \mathrm{mg} /$ $\mathrm{dL}$ were excluded from this study. Subjects taking anti-hypertensive, oral-hypoglycemic agents, insulin treatment, or lipid-lowering medications were also excluded. BMI was obtained by body weight $(\mathrm{kg})$ divided by squared body height $\left(\mathrm{m}^{2}\right)$. PBF was determined from bioelectrical impedance analyses (BIA) using TANITA TBF-215 (TANITA Corporation, Tokyo, Japan). This method measures the flow of electrical signals as they pass through fat and lean water in the body. When the amount of fat and lean matter or water changes, so do the signals, giving a reliable and accurate measurement of the amount of each of these components that make up the total weight of the person. The measurements were performed in a standing position, with electrodes in contact with the soles and heels of both feet. Serum total cholesterol (TC), triglycerides (TG), and high-density lipoprotein (HDL)-C levels were determined by standard enzymatic methods. LDL-C levels were calculated with the Friedewald formula. A statement of institutional approval of the study in accordance with the Declaration of Helsinki and informed consent were obtained from all of the participants in this study.

\section{Statistical Analysis}

Statistical evaluation was performed using StatView-J 5.0 software (SAS Institute, Cary NC on a Macintosh Computer).

The results were expressed as the mean \pm SD and the significance level was set at $p<0.05$. Pearson correlation coefficients were used to evaluate the relationship of age, BMI, PBF with metabolic parameters.

\section{Results}

\section{Relationship of PBF with BMI}

$\mathrm{PBF}$ had a strong correlation with BMI both in men $(r=0.813, p<0.0001)$ and women $(r=0.888, p$ $<0.0001)$.

\section{Relationship of PBF with Age}

PBF showed a weak inverse relationship with age in men $(\mathrm{r}=-0.149, p<0.0001)$, but had no significant relation with age in women $(\mathrm{r}=0.055, p=0.054)$.

\section{Relationships of Age, BMI and PBF with Several Metabolic Parameters in Men (Table 2)}

Age showed a positive relationship with sBP, while its relation with $\mathrm{dBP}$ was subtle. Both BMI and PBF showed positive relationships with sBP and $\mathrm{dBP}$. Age, BMI and PBF showed a weak association with FPG and HbA1C. There was no association of age with any of the lipid and lipoprotein parameters except for its subtle relation with Log TG. In contrast, both BMI and PBF were positively associated with TC, LDL-C and, to a higher degree, with log TG, while being inversely associated with HDL-C levels. 
Table 2. Correlation coefficient of age, BMI and PBF with metabolic parameters in men

\begin{tabular}{lccc}
\hline & age & BMI & PBF \\
\hline sBP & $0.235^{* * *}$ & $0.263^{* * *}$ & $0.21^{* * *}$ \\
dBP & $0.097^{* *}$ & $0.303^{* * *}$ & $0.271^{* * *}$ \\
fasting plasma glucose & $0.14^{* * *}$ & $0.178^{* * *}$ & $0.177^{* * *}$ \\
HbA1c & $0.22^{* * *}$ & $0.159^{* * *}$ & $0.152^{* * *}$ \\
total cholesterol & -0.002 & $0.171^{* * *}$ & $0.242^{* * *}$ \\
LDL-cholesterol & 0.033 & $0.201^{* * *}$ & $0.25^{* * *}$ \\
log-triglycerides & $-0.082^{*}$ & $0.337^{* * *}$ & $0.371^{* * *}$ \\
HDL-cholesterol & 0.009 & $-0.312^{* * *}$ & $-0.283^{* * *}$ \\
\hline
\end{tabular}

sBP, systolic blood pressure; dBP, diastolic blood pressure

*** $p<0.0001 ; * * p<0.001,{ }^{*} p<0.01$
Table 3. Correlation coefficient of age, BMI and PBF with metabolic parameters in women

\begin{tabular}{lccc}
\hline & age & BMI & PBF \\
\hline sBP & $0.379^{* * *}$ & $0.277^{* * *}$ & $0.254^{* * *}$ \\
dBP & $0.133^{* * *}$ & $0.159^{* * *}$ & $0.148^{* * *}$ \\
fasting plasma glucose & $0.289^{* * *}$ & $0.237^{* * *}$ & $0.223^{* * *}$ \\
HbA1c & $0.351^{* * *}$ & $0.21^{* * *}$ & $0.162^{* * *}$ \\
total cholesterol & $0.292^{* * *}$ & $0.102^{* *}$ & $0.158^{* * *}$ \\
LDL-cholesterol & $0.269^{* * *}$ & $0.179^{* * *}$ & $0.227^{* * *}$ \\
log-triglycerides & $0.244^{* * *}$ & $0.243^{* * *}$ & $0.258^{* * *}$ \\
HDL-cholesterol & -0.034 & $-0.287^{* * *}$ & $-0.266^{* * *}$ \\
\hline
\end{tabular}

sBP, systolic blood pressure; dBP, diastolic blood pressure *** $p<0.0001 ;{ }^{* *} p<0.001,{ }^{*} p<0.01$

Table 4. Multiple regression analysis on the relationship of age, BMI and PBF with metabolic parameters in men

\begin{tabular}{|c|c|c|c|c|c|c|c|c|c|}
\hline \multirow{2}{*}{ variables } & \multicolumn{3}{|c|}{ age } & \multicolumn{3}{|c|}{ BMI } & \multicolumn{3}{|c|}{$\mathrm{PBF}$} \\
\hline & $\beta$ & $\mathrm{t}$ & $p$ & $\beta$ & $\mathrm{t}$ & $p$ & $\beta$ & $\mathrm{t}$ & $p$ \\
\hline$s B P$ & 0.264 & 11.25 & $<0.0001$ & 0.227 & 5.725 & $<0.0001$ & 0.067 & 1.667 & 0.0957 \\
\hline $\mathrm{dBP}$ & 0.139 & 5.839 & $<0.0001$ & 0.231 & 5.74 & $<0.0001$ & 0.098 & 2.399 & 0.0165 \\
\hline FPG & 0.17 & 6.953 & $<0.0001$ & 0.078 & 1.873 & 0.0613 & 0.137 & 3.289 & 0.001 \\
\hline HbA1c & 0.256 & 10.62 & $<0.0001$ & 0.101 & 2.464 & 0.0138 & 0.091 & 2.198 & 0.0281 \\
\hline total cholesterol & 0.037 & 1.529 & 0.1264 & -0.049 & -1.197 & 0.2314 & 0.3 & 7.185 & $<0.0001$ \\
\hline LDL-cholesterol & 0.01 & 0.401 & 0.6884 & 0.014 & 0.33 & 0.7413 & 0.13 & 3.002 & 0.0027 \\
\hline Log triglycerides & -0.046 & -1.989 & 0.0468 & 0.135 & 3.421 & 0.0006 & 0.261 & 6.558 & $<0.0001$ \\
\hline HDL-cholesterol & -0.015 & -0.638 & 0.5239 & -0.235 & -5.804 & $<0.0001$ & -0.1 & -2.457 & 0.0141 \\
\hline
\end{tabular}

FPG, fasting plasma glucose

\section{Relationships of Age, BMI and PBF with Several Metabolic Parameters in Women (Table 3)}

Age, BMI and PBF showed positive associations with sBP and, to a lesser degree, with $\mathrm{dBP}$. In women, the association of age, BMI and PBF with FPG were higher and likewise those with HbAlc were higher than in men. Unlike in men, age had significant associations with serum TC, Log TG and LDL-C levels, but not with HDL-C levels. BMI showed a positive association with Log TG and, to a lesser degree, with TC and LDL-C, while showing an inverse relationship with serum HDL-C. Similarly, PBF showed a positive association with Log TG and an inverse one with HDL-C, and the associations of PBF with TC or LDL-C were relatively weak compared with those in men.

\section{Multiple Regression Analysis on the Relationships of Age, BMI and PBF with Several Metabolic Parameters in Men (Table 4) \\ Multiple regression analysis with BMI, age and}

$\mathrm{PBF}$ as independent variables and with several metabolic parameters as dependent variables showed that the relation of $\mathrm{PBF}$ with blood pressure was not as strong as age or BMI.

The relation of PBF with FPG and HbA1c was not as strong as age. Of note, the relation of PBF with TC and TG was more pronounced than BMI and age with these lipid parameters. In contrast, BMI was more strongly associated with serum HDL-C levels than PBF and age.

Multiple Regression Analysis on the Relationships of Age, BMI and PBF with Several Metabolic Parameters in Women (Table 5)

The relationship between blood pressure and PBF did not persist after adjustment for BMI and age. Similarly, the relationship between glucose metabolism parameters and PBF did not persist or almost disappeared after adjustment for other co-variants. In contrast, the relationship between PBF and TC, TG and LDL-C was stronger than those between BMI 
Table 5. Multiple regression analysis on the relationship of age, BMI and PBF with metabolic parameters in women

\begin{tabular}{|c|c|c|c|c|c|c|c|c|c|}
\hline variables & \multicolumn{3}{|c|}{ age } & \multicolumn{3}{|c|}{ BMI } & \multicolumn{3}{|c|}{ PBF } \\
\hline sBP & 0.367 & 13.96 & $<0.0001$ & 0.188 & 3.358 & 0.0008 & 0.062 & 1.104 & 0.2698 \\
\hline $\mathrm{dBP}$ & 0.118 & 4.204 & $<0.0001$ & 0.129 & 2.094 & 0.0364 & 0.033 & 0.535 & 0.5926 \\
\hline FPG & 0.264 & 9.792 & $<0.0001$ & 0.086 & 1.464 & 0.1436 & 0.116 & 1.976 & 0.0484 \\
\hline HbAlc & 0.326 & 12.337 & $<0.0001$ & 0.212 & 3.664 & 0.0003 & -0.036 & -0.617 & 0.5375 \\
\hline Log triglycerides & 0.229 & 8.54 & $<0.0001$ & 0.017 & 0.287 & 0.774 & 0.248 & 4.245 & $<0.0001$ \\
\hline HDL-cholesterol & -0.015 & -0.547 & 0.5844 & -0.221 & -3.676 & 0.0002 & -0.078 & -1.305 & 0.1921 \\
\hline
\end{tabular}

FPG, fasting plasma glucose

Table 6. Pearson's correlation coefficients between serum lipids vs. body mass index or percent body fat by gender and age

\begin{tabular}{|c|c|c|c|c|c|c|c|c|c|c|}
\hline age (y) & $\mathrm{n}$ & \multicolumn{4}{|c|}{ body mass index } & \multicolumn{4}{|c|}{ percent body fat } & $\mathrm{PBF}$ vs $\mathrm{BM}$ \\
\hline $30-39$ & 122 & 0.146 & $0.387^{* * *}$ & $-0.43^{* * *}$ & $0.246^{* *}$ & $0.25^{* *}$ & $0.417^{* * *}$ & $-0.383^{* * *}$ & $0.323^{* * *}$ & $0.858^{* * *}$ \\
\hline $40-49$ & 438 & $0.153^{* *}$ & $0.356^{* * *}$ & $-0.334 * * *$ & $0.182^{* * *}$ & $0.236^{* * *}$ & $0.348^{* * *}$ & $-0.247 * * *$ & $0.238^{* * *}$ & $0.817^{* * *}$ \\
\hline $50-59$ & 477 & $0.209^{* *}$ & $0.309^{* * *}$ & $-0.289^{* * *}$ & $0.225^{* * *}$ & $0.269^{* * *}$ & $0.396^{* * *}$ & $-0.314^{* * *}$ & $0.265^{* * *}$ & $0.822^{* * *}$ \\
\hline $70-79$ & 67 & 0.136 & $0.327^{* *}$ & -0.144 & 0.125 & 0.133 & $0.296^{*}$ & -0.165 & 0.148 & $0.699^{* * *}$ \\
\hline \multicolumn{11}{|l|}{ women } \\
\hline $30-39$ & 86 & -0.04 & $0.317^{* *}$ & $-0.414^{* * *}$ & 0.07 & 0.056 & $0.342^{* * *}$ & $-0.344^{* *}$ & 0.148 & $0.917^{* * *}$ \\
\hline $40-49$ & 355 & $0.134^{*}$ & $0.273^{* * *}$ & $-0.306^{* * *}$ & $0.228^{* * *}$ & $0.211^{* *}$ & $0.321^{* * *}$ & $-0.278^{* * *}$ & $0.279^{* * *}$ & $0.876^{* * *}$ \\
\hline $50-59$ & 408 & 0.081 & $0.212^{* * *}$ & $-0.263^{* * *}$ & $0.141^{* *}$ & $0.178^{* *}$ & $0.238^{* * *}$ & $-0.236^{* * *}$ & $0.23^{* * *}$ & $0.885^{* * *}$ \\
\hline
\end{tabular}

${ }^{*} p<0.05, * * p<0.01, * * * p<0.001$

TC, total cholesterol; TG, triglycerides; PBF, percent body fat; BMI, body mass index

and these lipid parameters. However, the association between PBF and HDL-C did not persist after adjustment for BMI and age, while in contrast, BMI was the independent determinant of HDL-C levels among age, BMI and PBF.

\section{Relationships of BMI or PBF with Several Metabolic Parameters After Subclassifying the Subjects According to Age Group (Table 6)}

In men, PBF had better associations with LDL-C across all ages and with TG in middle age than did BMI. In women, PBF had better associations with LDL-C and TG than did BMI across the age. In contrast, $\mathrm{PBF}$ had weaker associations than BMI with HDL-C in young to middle aged men and women. Also, the relation between PBF and BMI was weaker in the elderly population.

\section{Discussion}

In this study, we analyzed the relationships of PBF by BIA with several metabolic parameters in 1,380 men and 1,103 women who underwent medical checkups from 1999-2002. Our findings are consistent with a previous study that compared with BMI. PBF by BIA was more strongly correlated with serum lipids except for HDL-C in Japanese population ${ }^{8)}$, although unlike our study they did not present data on PG and BP. We also subclassified the subjects according to age and measured Pearson's correlation coefficients between lipids and BMI or PBF by gender and age.

Despite PBF measured by BIA being a simple, 
convenient and popular way of assessing body fat in daily clinical practice ${ }^{1-3)}$, to our knowledge its clinical significance has not been well clarified. This is in stark contrast to the BMI, which has long been recognized as a predictor of morbidity and mortality due to numerous chronic diseases, including type 2 diabetes, cardiovascular disease and stroke, ${ }^{9,10}$.

Obesity is determined based on an individual's BMI, which just like PBF, does not provide any information on an individual's body fat distribution but is the widely standardized parameter for defining obesity. Indeed, in this study, we found that this parameter was a good predictor of BP, especially for men. PBF, on the other hand, was not likely to be a good predictor of BP in both genders. Likewise, it was not a good predictor of an individual's fasting glucose and HbA1c levels in both genders. In contrast, it was found to be the strongest predictor of TC, LDL-C and TG among age, BMI and PBF in men. Although the mechanism of TG's association with PBF has not been elucidated, we presume that it is highly related to the fact that adipose tissue is the main store of TG in the body. TG in adipose tissue undergoes hydrolysis by a hormone-sensitive lipase to form free fatty acids. The liver in turn takes up free fatty acids from the circulation and cause the formation and secretion of VLDL ${ }^{11)}$. VLDL is subsequently metabolized into IDL, leading to the formation of LDL. Among the lipid parameters we investigated, unlike TC, TG or LDL-C, HDL-C was much more closely associated with BMI than was PBF. We speculate the potential reasons for this as follows: HDL-C is first secreted by the liver and intestine as small, lipid poor, apo A-1 particles termed nascent $\mathrm{HDL}^{12)}$. These HDL particles interact with the ATPbinding cassette transport protein $\mathrm{A} 1$ ( $\mathrm{ABC} \mathrm{A} 1$ ) on peripheral cells such as arterial wall macrophages to drive cholesterol efflux ${ }^{13,14)}$. This step is followed by the maturation of HDL particles by the function of lecithin-cholesterol acyltransferase (LCAT) ${ }^{15,16)}$. Cholesteryl ester transfer protein, which is produced in the liver, spleen, skeletal muscle and adipocytes, is involved in cholesteryl ester transfer from HDL particles to apoB-containing particles, including very low density lipoprotein (VLDL) and LDL ${ }^{17}, 18$. . Hepatic lipase (HL), which is mainly produced in the liver, also plays a key role in the metabolism of HDL particles ${ }^{19,20)}$. Specifically, HL hydrolyzes TG in HDL, generating small-modified HDL particles, which are taken up primarily by the scavenger receptor class $\mathrm{B}$ type I on the surface of hepatocytes ${ }^{21,22)}$. These lots of steps for determining serum HDL concentration might account for the relatively weak association of HDL-C with PBF
In both sexes, but especially in men, young subjects had closer associations between BMI and PBF than did elder individuals, and these associations slightly declined with age, which may be due to increased in inter-individual variations of body composition with age $^{23)}$, since body composition may be affected by many age-related changes, such as decreases in bone, muscle, and body water, and distribution of fat ${ }^{24,25)}$. Also, it has been shown that PBF measured by BIA is less precise and less accurate in the elderly than in young individuals ${ }^{26}$.

Indeed, in our study, the degree of the association of lipids to BMI or PBF also changed according to age.

Besides the method studied in this study, recently, an excellent and sophisticated technique by BIA was introduced ${ }^{27)}$, which appears to be able to provide us with information on visceral fat mass without using CT. Given that the importance of measuring visceral fat, not whole body fat, has been increasingly emphasized recently ${ }^{28)}$, we hope that this method will prevail in the near future.

Recently, more and more attention has been given to the measurement of waist circumference, because it has now become the essential component for diagnosing metabolic syndrome. Our previous study has shown that PBF versus BMI was highly related to waist circumference in middle-aged men $([\mathrm{r}=0.893, p<$ $0.0001]$ versus $[\mathrm{r}=0.876, p<0.0001])$ in the Kanazawa district ${ }^{29)}$.

In conclusion, based on our findings of 1,380 male and 1,103 female Japanese healthy subjects, we suggest that measuring percent body fat by bioelectrical impedance analyses (BIA) may be superior to BMI for predicting an individual's serum lipids except for HDL-C in middle-aged men and women.

\section{References}

1) Lukaski HC, Johnson PE, Bolonchuk WW, and Lykken GI: Assessment of fat-free mass using bioelectrical impedance measurements of the human body. Am J Clin Nutr, $1985 ; 41: 810-817$

2) Tsui EY, Gao XJ, and Zinman B: Bioelectrical impedance analysis (BIA) using bipolar foot electrodes in the assessment of body composition in Type 2 diabetes mellitus. Diabet Med, 1998; 15:125-128

3) Singh RB, Niaz MA, Beegom R, Wander GS, Thakur AS, and Rissam HS: Body fat percent by bioelectrical impedance analysis and risk of coronary artery disease among urban men with low rates of obesity: the Indian paradox. J Am Coll Nutr, 1999; 18:268-273

4) Fujioka S, Matsuzawa Y, Tokunaga K, and Tarui S: Contribution of intra-abdominal fat accumulation to the impairment of glucose and lipid metabolism in human obe- 
sity. Metabolism, 1987; 36:54-59

5) Tokunaga K, Matsuzawa Y, Ishikawa K, and Tarui S: A novel technique for the determination of body fat by computed tomography. Int J Obes, 1983; 7:437-445

6) Ross R, Shaw KD, Martel Y, de Guise J, and Avruch L: Adipose tissue distribution measured by magnetic resonance imaging in obese women. Am J Clin Nutr, 1993; $57: 470-475$

7) Suzuki R, Watanabe S, Hirai Y, Akiyama K, Nishide T, Matsushima Y, Murayama H, Ohshima H, Shinomiya M, Shirai K, et al: Abdominal wall fat index, estimated by ultrasonography, for assessment of the ratio of visceral fat to subcutaneous fat in the abdomen. m J Med, 1993; 95:309314

8) Nagaya T, Yoshida H, Takahashi H, Matsuda Y, and Kawai M: Body mass index (weight/height2) or percentage body fat by bioelectrical impedance analysis: which variable better reflects serum lipid profile? nt J Obes Relat Metab Disord, 1999; 23:771-774

9) World Health Organization. Obesity: preventing and managing the global epidemic. Report of a WHO consultation on obesity. Geneva: World Health Organization, 1998

10) National Institutes of Health, National Heart, Lung, and Blood Instutes. Clinical Guidelines on the Identification, Evaluation, and Treatment of Overweight and Obesity in Adults-The Evidence Report. Obes Res, 1998; 6:S51-210

11) Kane JP and Havel RJ: Disorders of the biosynthesis and secretion of lipoproteins containing the B apolipoproteins. In: The Metabolic \& Molecular Basis of Inherited Disease. 8th Ed, ed by Scriver CR, Beaudet AL, Sly WS, Valle D, pp2717-2752, McGraw-Hill, New York, 2001

12) Gotto AM Jr. and Brinton EA: Assessing low levels of high-density lipoprotein cholesterol as a risk factor in coronary heart disease: a working group report and update. J Am Coll Cardiol, 2004; 43:717-724

13) Marcil M, Brooks-Wilson A, Clee SM, Roomp K, Zhang LH, Yu L, Collins JA, van Dam M, Molhuizen HO, Loubster O, Ouellette BF, Sensen CW, Fichter K, Mott S, Denis M, Boucher B, Pimstone S, Genest J Jr., Kastelein $\mathrm{JJ}$, and Hayden MR: Mutations in the ABC1 gene in familial HDL deficiency with defective cholesterol efflux. Lancet, 1999; 354:1341-1346

14) Bodzioch M, Orso E, Klucken J, Langmann T, Bottcher A, Diederich W, Drobnik W, Barlage S, Buchler C, PorschOzcurumez M, Kaminski WE, Hahmann HW, Oette K, Rothe G, Aslanidis C, Lackner KJ, and Schmitz G: The gene encoding ATP-binding cassette transporter 1 is mutated in Tangier disease. Nat Genet, 1999; 22:347-351

15) Jonas A: Lecithin cholesterol acyltransferase. Biochim Biophys Acta, 1999; 1529:245-256

16) Santamarina-Fojo S, Lambert G, Hoeg JM, and Brewer
HB Jr.: Lecithin-cholesterol acyltransferase: role in lipoprotein metabolism, reverse cholesterol transport and atherosclerosis. Curr Opin Lipidol, 2000; 11:267-275

17) Inazu A, Koizumi J, and Mabuchi H: Cholesteryl ester transfer protein and atherosclerosis. Curr Opin Lipidol, 2000; 11:389-396

18) Barter PJ and Rye KA: Cholesteryl ester transfer protein, high density lipoprotein and arterial disease. Curr Opin Lipidol, 2001; 12:377-382

19) Santamarina-Fojo S, Gonzalez-Navarro H, Freeman L, Wagner E, and Nong Z: Hepatic lipase, lipoprotein metabolism, and atherogenesis. Arterioscler Thromb Vasc Biol, 2004; 24:1750-1754

20) Thuren T: Hepatic lipase and HDL metabolism. Curr Opin Lipidol, 2000; 11:277-283

21) Acton S, Rigotti A, Landschulz KT, Xu S, Hobbs HH, and Krieger M: Identification of scavenger receptor SR$\mathrm{BI}$ as a high density lipoprotein receptor. Science, 1996; 271:518-520

22) Krieger M: Charting the fate of the "good cholesterol": identification and characterization of the high-density lipoprotein receptor SR-BI. Annu Rev Biochem, 1999; 68:523-558

23) Carmichael CM and McGue M: A cross-sectional examination of height, weight, and body mass index in adult twins. J Gerontol A Biol Sci Med Sci, 1995; 50:B237-244

24) Baumgartner RN, Stauber PM, McHugh D, Koehler KM, and Garry PJ: Cross-sectional age differences in body composition in persons $60+$ years of age. J Gerontol A Biol Sci Med Sci, 1995; 50:M307-316

25) Baumgartner RN: Body composition in elderly persons: a critical review of needs and methods. Prog Food Nutr Sci, 1993; 17:223-260

26) Roubenoff R, Dallal GE, and Wilson PW: Predicting body fatness: the body mass index vs estimation by bioelectrical impedance. Am J Public Health, 1995; 85:726-728

27) Ryo M, Maeda K, Onda T, Katashima M, Okumiya A, Nishida M, Yamaguchi T, Funahashi T, Matsuzawa Y, Nakamura $\mathrm{T}$, and Shimomura I: A new simple method for the measurement of visceral fat accumulation by bioelectrical impedance. Diabetes Care, 2005; 28:451-453

28) Carr DB, Utzschneider KM, Hull RL, Kodama K, Retzlaff BM, Brunzell JD, Shofer JB, Fish BE, Knopp RH, and Kahn SE: Intra-abdominal fat is a major determinant of the National Cholesterol Education Program Adult Treatment Panel III criteria for the metabolic syndrome. Diabetes, 2004; 53:2087-2094

29) Kobayashi J, Murase Y, Asano A, Nohara A, Kawashiri MA, Inazu A, Yamagishi M, and Mabuchi H: Effects of Walking with a Pedometer on Serum Lipid and Adiponectin Levels in Japanese Middle-aged Men. J Atheroscler Thromb, 2006; 13:197-201 